\title{
Transitioning ifosfamide chemotherapy regimens to the ambulatory setting: reviewing cost savings and safety profile
}

\author{
Cindy Banh ${ }^{1} \cdot$ Kendall Valsvik $^{1} \cdot$ Alejandra Arredondo ${ }^{1} \cdot$ Kassie Notbohm $^{1} \cdot$ Emad Elquza $^{1} \cdot$ Hani Babiker $^{1}$. \\ Andrew $\mathrm{Kraft}^{1} \cdot$ Alejandro Recio Boiles $^{1} \cdot$ Daniel Persky ${ }^{1} \cdot$ Alicia Ortega $^{1} \cdot$ Ali McBride $^{1} \mathbb{C}$
}

Received: 6 March 2021 / Accepted: 24 October 2021 / Published online: 26 November 2021

(c) The Author(s), under exclusive licence to Springer-Verlag GmbH Germany, part of Springer Nature 2021

\begin{abstract}
Purpose To characterize and compare both the outcome and cost of treatment of outpatient (OP) and inpatient (IP) ifosfamide therapy.

Methods A single-center retrospective chart review of patients 18 years and older receiving ifosfamide therapy. The primary endpoint compares and evaluates the side effect profiles of ifosfamide-treated patients in the OP/IP settings. The adverse event grading system was characterized using the CTCAE Version 5.0. The highest grade was documented per cycle. The secondary endpoint of this study compares the costs of OP/IP therapy. It was assumed that the cost of medication was equivalent for IP/ OP treatments. The cost saved with OP administration was determined by the average cost of hospital stay for IP admission. Results Ifosfamide therapy of 86 patients (57 OP, 29 IP) was reviewed. The predominant OP regimens were doxorobucinifosfamide-mesna (AIM) with $43.9 \%$ and ifosfamide-etoposide (IE) with $29.8 \%$. Grade 4 anemia, thrombocytopenia, and neutropenia were most frequent in IP vs OP therapies (22.9\% IP vs $4.3 \%$ OP, $21.6 \%$ IP vs $9.2 \%$ OP, and $22.8 \%$ IP vs $19.6 \%$ OP respectively). Neutropenic fever (NF) occurred in 20 OP patients which were predominantly treated with AIM or IE and led to average hospital stay of 6 days. Neurotoxicity, treated with methylene blue (MB) occurred in 4 OP patients. OP therapy saved a total of 783 hospital days, leading to a cost savings of $\$ 2,103,921$.

Conclusions Transitioning ifosfamide to the OP setting is feasible for academic and community infusion centers with the OP administration being safe, well-tolerated, and associated with decreased total cost of care. The current processes allow for safe transition of chemotherapy of chemotherapy under times of COVID.
\end{abstract}

Keywords Ifosfamide $\cdot$ Outpatient chemotherapy $\cdot$ Alternative payment model $\cdot$ Supportive care $\cdot$ Oncology care model

\section{Introduction}

Over the last three decades, there has been a large emergence of new treatment regimens and combination treatments in chemotherapy; many of these combinations are aggressive and have led to hospital admissions secondary to acute toxicities exhibited during treatment. Due to these complications, monitoring procedures were developed for acute supportive care models for treatment [1]. Treatment regimens or therapies are often administered IP based on select criteria:

Ali McBride

mcbride@pharmacy.arizona.edu

1 The University of Arizona Cancer Center, Tucson, AZ, USA
- Highly chemo-toxic therapies such as high dose cisplatin, high-dose methotrexate

- Medical procedures requiring acute care: intra-arterial chemotherapy, acute leukemic inductions, medically emergent rituximab

- Required close monitoring and observation: emergent chemotherapy, high-risk cytokine release syndrome therapies, desensitization protocols

- Complex chemotherapy regimens, which require six or more hours of observation and/or drug administration [2]

In regard to these criteria, the use of IP treatment is required for patient welfare and care.

The cost of cancer therapies has also increased over the last two decades with increased costs of care and integration of new, novel agents [3]. Recent reports have demonstrated chemotherapy treatments are continuing to increase annually 
at exponential rates. In the USA, the total expenditures for chemotherapy agents increased from $\$ 26.8$ billion in 2011 to $\$ 42.1$ billion in 2016 . The largest increase seen was between 2015 and 2016 with an increase of $15.6 \%$ [4]. These costs have led to higher financial costs for both institution and the patient, which impacts the quality of life of cancer patients [5-7].

Due to higher costs of therapies in the inpatient (IP) setting and implementation of value-based performance measures with new alternative payment models, new approaches to patient care are being addressed to optimize therapies for patients. The transition from administering IP chemotherapy to the outpatient (OP) setting provides a unique opportunity to decrease total patient care costs, improve patient quality of life, and also maximize patient assistance programs that would not otherwise be accessible to patients being treated IP. Ideally, transitioning to the ambulatory setting would save on hospital stay days and possibly on the infusion drug being administered. For example, regimens containing ifosfamide are normally administered IP, namely for side effect monitoring and days of infusion needed. Currently, certain ifosfamide-based regimens were moved to the OP setting at the University of Arizona Cancer Center to enhance patient care. The transition was evaluated by addressing factors requiring hospitalizations, clinical parameters, caregiver support, observation and mitigation, and the management of side effects for OP treatment [1].

Ifosfamide is an alkylating agent that has activity in several cancer disease states, including germ cell tumors, soft tissue sarcomas, and lymphomas [8-10]. It is used in several oncology regimens and commonly administered in the IP setting due to monitoring requirements and side effect management [11]. Due to an acrolein metabolite, ifosfamide treatment can cause toxicity of urinary epithelium potentiating for hemorrhagic cystitis. This adverse effect is mitigated with mesna, a chemoprotective agent [12]. Infusion centers may send patients home with a 24-h or longer infusion pump for intravenous administration of mesna at home.

Currently, there is limited data to validate the safety and potential cost-savings of transitioning ifosfamide regimens to the ambulatory setting. In 2009 at a single ambulatory setting, a study of 13 patients given monotherapy ifosfamide plus mesna showed the feasibility, safety, and healthcare cost saving of OP treatment [13]. Even though the concept is a decade old, ambulatory chemotherapy for ifosfamide is still in its infancy [1, 13]. With new measures set forth for the response to the COVID-19 pandemic, more centers have adapted to OP treatment in an attempt to decrease the risk of coronavirus transmission to cancer patients in the IP setting [14].

The purpose of this study was to characterize the side effect profile of ifosfamide therapy in the OP compared to IP treatment regimens. The IP and OP treatment costs were based on the costs of hospital stay per day for therapy administration. Costs of CBC/CMP lab orders between IP and OP regimens were also included. Observational comparisons were made between adverse reactions (ADRs) and the costs of therapy in either treatment regimen The University of Arizona Cancer Center.

\section{Methods}

\section{Patient eligibility}

This was a single-center retrospective chart review to characterize and compare outcomes and cost savings between patients receiving IP and OP ifosfamide treatment. This study was approved by the University of Arizona Human Subjects Protection Program. The primary outcome was the characterized side effect profile and safety of ifosfamide treatment in both the OP and IP settings. The adverse event grading system was characterized using the CTCAE Version 5.0 [15]. If a patient chart documented a side effect with varying severity throughout a single cycle, the highest grade achieved was recorded for that cycle. Secondary endpoints included the number of hospitalizations and the cost of ifosfamide treatment. The different ifosfamide chemotherapy regimens observed included doxorubicin-ifosfamide-mesna (AIM), ifosfamide-etoposide (IE), paclitaxel-ifosfamidecisplatin (TIP), etoposide-ifosfamide-cisplatin (VIP), and others as specified in Table 1. The inclusion criteria for this study included patients 18 years and older who received ifosfamide therapy in the OP and/or IP setting at the University of Arizona Cancer Center between September 1, 2013 through August 31, 2019.

\section{Data collection}

Data was collected electronically using Excel data collection sheets on password-protected computers at the University of Arizona Cancer Center. Demographic data and descriptive information were collected on gender, age, ethnicity, weight, body mass index (BMI), zip code, type of cancer, number of treatment cycles of ifosfamide therapy, type/ severity of adverse effects, number of hospitalizations, and cost of treatment.

\section{Cost analysis}

The cost saved with OP administration of ifosfamide was determined by the average cost of hospital stay for IP admission and cost of lab orders assuming the cost of the medication was the same for both IP and OP treatments. The expense per IP day used for this analysis was \$2687 and was based on an adjusted hospital expense indicator for the state 
Table 1 Chemotherapy orders and number of patients

\begin{tabular}{|c|c|c|c|c|}
\hline $\begin{array}{l}\text { Cancer-based } \\
\text { chemotherapy } \\
\text { treatment }\end{array}$ & Outpatient $^{\mathrm{a}}$ & Total cycles & Inpatient $^{\mathrm{a}}$ & Total cycles \\
\hline \multicolumn{5}{|l|}{ Sarcoma } \\
\hline AIM & 25 & 92 & 3 & 5 \\
\hline IE & 17 & 42 & 1 & 1 \\
\hline Carbo/Doxo/Ifos & 0 & 0 & 1 & 6 \\
\hline $\begin{array}{l}\text { AEWS } \\
1031 / 1221\end{array}$ & 2 & 7 & 0 & 0 \\
\hline \multicolumn{5}{|l|}{ Germ cell tumors } \\
\hline TIP & 4 & 7 & 2 & 7 \\
\hline VIP & 6 & 11 & 4 & 13 \\
\hline ACNS 1123 & 0 & 0 & 1 & 3 \\
\hline TI & 2 & 11 & 1 & 2 \\
\hline \multicolumn{5}{|l|}{ Lymphoma } \\
\hline IGEV & 1 & 2 & 0 & 0 \\
\hline ICE & 0 & 0 & 7 & 12 \\
\hline R-ICE & 4 & 10 & 8 & 22 \\
\hline IVAC & 0 & 0 & 1 & 2 \\
\hline SMILE & 0 & 0 & 1 & 2 \\
\hline \multicolumn{5}{|l|}{ Lung cancer } \\
\hline $\begin{array}{l}\text { Ifosfamide } \\
\text { (alone) }\end{array}$ & 4 & 7 & 0 & 0 \\
\hline
\end{tabular}

AIM doxorubicin, ifosfamide, mesna, $I E$ ifosfamide, etoposide, TIP paclitaxel, ifosfamide, cisplatin, VIP etoposide, ifosfamide, cisplatin, $I G E V$ ifosfamide, gemcitabine, vinorelbine, ICE ifosfamide, carboplatin, etoposide, R-ICE rituximab, ifosfamide, carboplatin, etoposide, IVAC ifosfamide, etoposide, cytarabine, SMILE dexamethasone, methotrexate, leucovorine, asparapinase, etoposide, AEWS 1031/1221 vincristine, doxorubicin, cyclophosphamide, ifosfamide, etoposide, ACNS1123 carboplatin, etoposide, ifosfamide, TI paclitaxel, ifosfamide ${ }^{a}$ Some patients had alternating ifosfamide containing regimens

of Arizona in 2018 [16]. Complete blood counts (CBCs) and comprehensive metabolic panel (CMP) labs were ordered together with a total cost of $\$ 96$ per order set [17]. Cost savings produced by changes in the tests ordered were calculated by comparing the difference in the average number of test sets ordered per cycle of IP and OP therapies.

\section{Results}

A total of 86 patients (57 OP, 29 IP) received at least one cycle of infusional ifosfamide between September 2013 and August 2019. The median patient age of the OP and IP regimens was 54 years (range 19-76) and 40.5 years (range 19-75), respectively. Males were the most predominant sex in both patient groups (68.4\% OP, 62\% IP). The population majority in both arms was white $(54.4 \%$ OP, $58.6 \%$ IP). Distance traveled for treatment was also evaluated in which $66.7 \%$ of the IP group and $58.6 \%$ of the OP group lived within 25 miles of the treatment institution as shown in Table 2.

For the OP chemotherapy regimens, the predominant diagnosis was sarcoma subtypes which was treated with AIM (43.9\%) or IE (29.8\%) regimens. There were 5 IP regimens (Carbo/Doxo/Ifos, ACNS 1123, ICE, IVAC, and SMILE) not given to OP patients and 3 OP regimens (AEWS $1031 / 1221$, IGEV, ifosfamide alone) not received by the other arm. Detailed demographics and the OP chemotherapy used in this data set are summarized in Tables 1 and 2 .

The total number of OP and IP cycles of treatment administered was 184 and 74, respectively, with an average of 3.2 OP cycles and 2.5 IP cycles per patient. Not all chemotherapy regimens had the same length of treatment days per cycle. Adverse drug effects and toxicities with their greatest severities were reported per cycle administered. Based on overall cycles including ifosfamide, anemia was the most common adverse reaction seen in both OP and IP regimens as seen in Fig. 1. Hematuria occurred in $2.7 \%$ of OP regimens and $4.1 \%$ of IP regimens, most commonly documented within the first cycle of treatment. OP regimens where hematuria occurred despite appropriate mesna therapy included AIM ( $n=1$, in cycle 1 and 2$)$ and one patient each in TIP (cycle 1), TI (cycle 7), and VIP (cycle 3). IP regimens with hematuria included one patient in both IVAC (cycle 1) and VIP (cycle 1 and 2).

Notable graded side effects were anemia, thrombocytopenia, and neutropenia. The incidence of grade 4 anemia was less frequently seen in OP therapy occurring in $4.3 \%$ of cycles ( $n=8$ cycles) compared to the IP therapy at $22.9 \%$ ( $n=17$ cycles). OP AIM treatment resulted in the most grade 4 anemia incidences $(n=4$ among 4 patients, two in both cycle 2 or 6 ), followed by AEWS1031 ( $n=2$ among 1 patient, in cycles 3 and 5) and IE ( $n=1$, in cycle 1$)$; whereas IP RICE treatment had the most grade 4 anemia cases $(n=5$ among 3 patients, in cycles $1-4$ with 2 in cycle 2 ), followed by TIP ( $n=3$ among 2 patients, twice in cycle 2 and cycle 3 ) and ICE ( $n=3$ among 3 patients, all cycle 1$)$. Grade 4 thrombocytopenia was more prevalent in IP therapies than OP at $21.6 \%$ of cycles $(n=16)$ vs $9.2 \%(n=17)$, respectively. The most OP cases occurred in EI treatment ( $n=6$ among 4 patients, two in cycles 1 and 4, once in cycles 2 and 7), followed by AIM ( $n=5$ among 4 patients, two in cycles 1 and 2 , once in cycle 6). The highest IP incidences occurred in Carb/Doxo/Ifos ( $n=5$ among 1 patient, cycles $1-5$ ), followed by RICE ( $n=4$ among 2 patients, cycles $1-4)$ and ICE ( $n=3$ among 3 patients, all cycle 1). Grade 4 neutropenia was also more prevalent in IP cycles at $22.8 \%(n=25)$ vs the $19.6 \%$ $(n=36)$ of OP cycles. The most OP incidences occurred in AIM $(n=14)$, IE $(n=10)$ and AEWS1031 $(n=6)$, whereas the most IP incidences were in RICE $(n=8)$, Carb/Doxo/ Ifos $(n=5)$, and AIM $(n=3)$. A complete list of OP and IP regimens and cycles where grade 4 neutropenia occurred 
Table 2 Patient demographics

\begin{tabular}{|c|c|c|}
\hline Patient demographics & Outpatient $(n=57)$ & Inpatient $(n=29)$ \\
\hline Male sex & $39(68.4 \%)$ & $18(62 \%)$ \\
\hline Age, median (range) & $54(19-76)$ & $40.5(19-75)$ \\
\hline \multicolumn{3}{|l|}{ Ethnicity } \\
\hline White & $31(54.4 \%)$ & $17(58.6 \%)$ \\
\hline Black & $0(0 \%)$ & $1(3.4 \%)$ \\
\hline Hispanic & $13(22.8 \%)$ & $6(20.7 \%)$ \\
\hline Asian & $2(3.5 \%)$ & $0(0 \%)$ \\
\hline Native American & $0(0 \%)$ & $2(6.9 \%)$ \\
\hline Non-specified & $11(19.3 \%)$ & $3(10.3 \%)$ \\
\hline \multicolumn{3}{|l|}{ BMI } \\
\hline Less than $18 \mathrm{~kg} / \mathrm{m}^{2}$ & $1(1.7 \%)$ & $0(0 \%)$ \\
\hline $18-25 \mathrm{~kg} / \mathrm{m} 2$ & $31(54.4 \%)$ & $13(44.8 \%)$ \\
\hline Greater than $25 \mathrm{~kg} / \mathrm{m}^{2}$ & $25(43.9 \%)$ & $16(55.2 \%)$ \\
\hline \multicolumn{3}{|l|}{ Malignancies } \\
\hline Breast cancer/HR +/ HER2 - /lung mets & $1(1.8 \%)$ & $0(0 \%)$ \\
\hline Germ cell & $4(7 \%)$ & $5(17.2 \%)$ \\
\hline Lung cancer & $5(8.8 \%)$ & $0(0 \%)$ \\
\hline Lymphoma & $7(12.3 \%)$ & $18(62 \%)$ \\
\hline Metastatic sarcoma to lung & $1(1.8 \%)$ & $0(0 \%)$ \\
\hline Osteosarcoma & $3(5.2 \%)$ & $2(7 \%)$ \\
\hline Penile cancer & $2(3.5 \%)$ & $1(3.4 \%)$ \\
\hline primitive neuroectodermal tumor & $1(1.8 \%)$ & $0(0 \%)$ \\
\hline Sarcoma & $5(8.8 \%)$ & $1(3.4 \%)$ \\
\hline Soft tissue sarcoma & $28(49 \%)$ & $2(7 \%)$ \\
\hline \multicolumn{3}{|l|}{ Cycles completed } \\
\hline 1 & 12 & 7 \\
\hline 2 & 20 & 10 \\
\hline 3 & 12 & $7 *$ \\
\hline 4 & 2 & $3 *$ \\
\hline 5 & 3 & 2 \\
\hline 6 & 8 & 1 \\
\hline Over 6 & 3 & 0 \\
\hline \multicolumn{3}{|l|}{ Distance traveled (mi) for treatment $* *$} \\
\hline $0-25$ & $38(66.7 \%)$ & $16(55.2 \%)$ \\
\hline $26-50$ & $3(5.3 \%)$ & $2(6.9 \%)$ \\
\hline $51-100$ & $10(17.5 \%)$ & $3(10.3 \%)$ \\
\hline $101-250$ & $2(3.5 \%)$ & $5(17.2 \%)$ \\
\hline $251-400$ & $2(3.5 \%)$ & $0(0 \% \%)$ \\
\hline$>400$ & $2(3.5 \%)$ & $2(6.9 \%)$ \\
\hline
\end{tabular}

*There was one IP patient who underwent two different ifosfamide regimens, completing a 3 cycle and 4 cycle course

** One IP patient's zip code was not available during chart review

is found in Table 3. Other adverse effects and toxicities are detailed in Figs. 1 and 2a, b. Additionally, graded side effects per regimen can be found in the Appendix Table 5 and 6.

Neutropenic fever (NF) was seen in 20 OP ifosfamide cycles $(10.9 \%)$ and 10 IP cycles $(13.5 \%)$. Of the 20 incidents of NF in the OP group, the predominant regimen administered was AIM $(n=10)$, followed by IE $(n=4)$ and VIP $(n=3)$ (Table 4$)$. NF was most commonly seen during administration of cycles 1 and 2 of OP therapy, leading to 19
(95\%) hospitalizations with an average length of stay (LOS) of 6 days (range $1-17$ days). Thirty percent $(n=6)$ of NF hospitalizations showed positive cultures (Table 4).

Neurotoxicity was seen in 4 patients with OP regimens: 3 patients in AIM and 1 in IE. These events took place within the first or second cycle of therapy, proportionally. All patients received MB to treat their neurologic syndrome. The mental status of one patient improved after $24 \mathrm{~h}$ on MB. Only one patient was 
Fig. 1 Frequency of OP and IP ifosfamide therapy adverse events for total cycles administered $(n=184$ OP cycles, $n=74$ IP cycles)

\section{Inpatient and Outpatient Adverse Events}

45

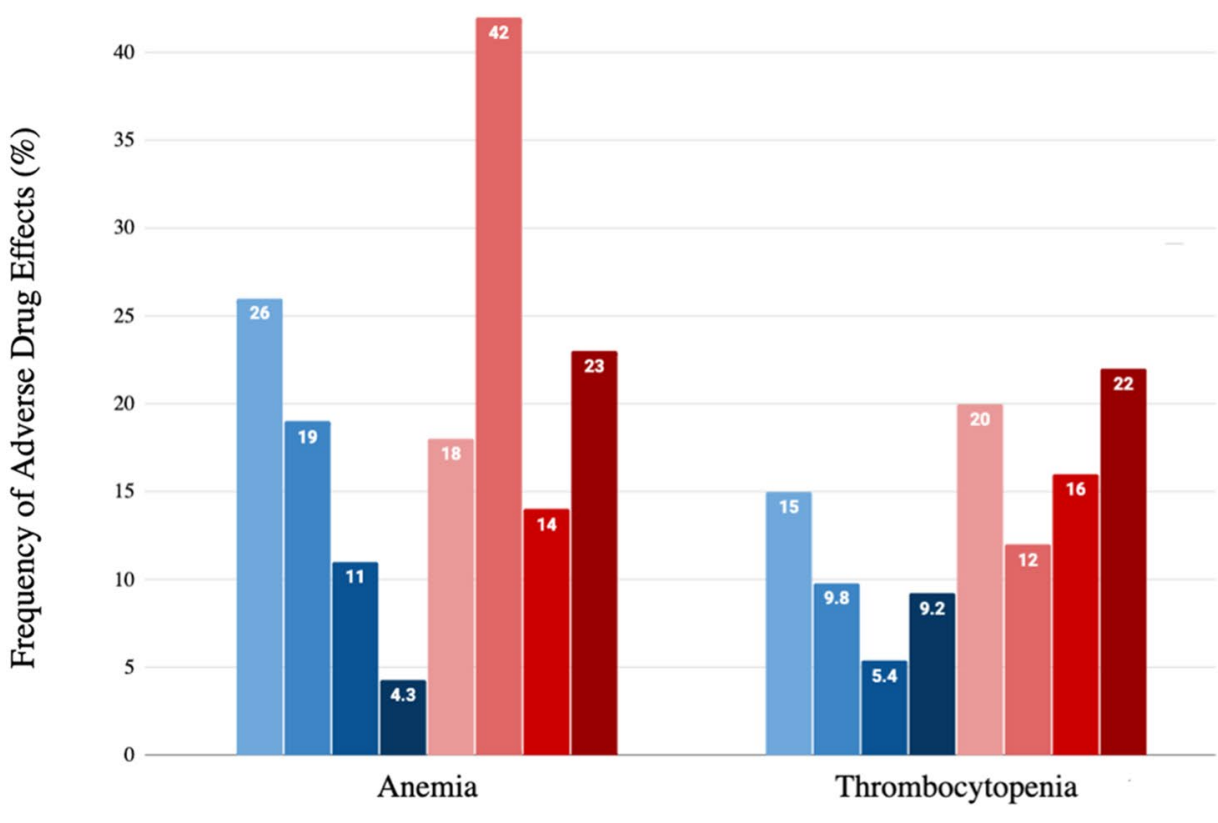

45

40

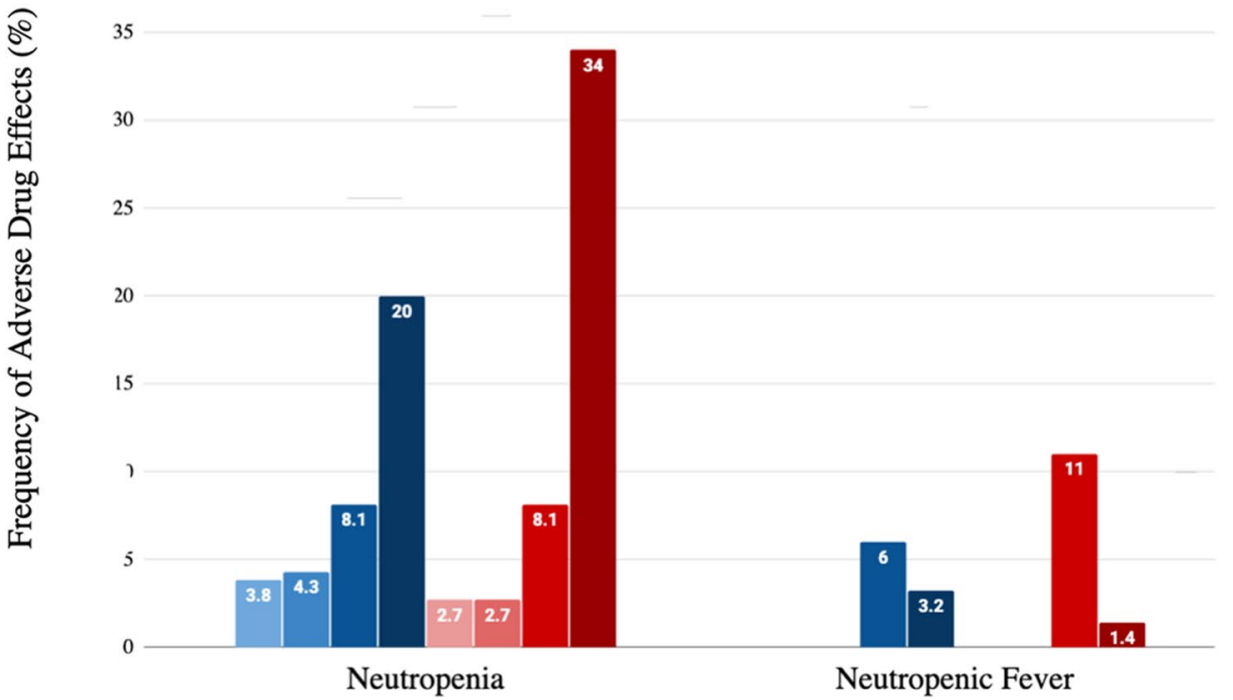

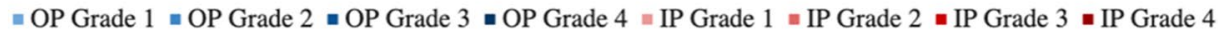

rechallenged with AIM treatment which contained a lower ifosfamide dose and shorter course. Neurotoxicity was seen in 3 patients with IP regimens including one for each AIM, ICE, and RICE therapies. All 3 incidents occurred during cycle 1 of therapy. Two patients were given $\mathrm{MB}$, and therapy was discontinued or changed to an alternative regimen.
A total of 36 patients (61\%) receiving outpatient chemotherapy were hospitalized at least once. Hospitalization was most common in patients who received AIM, with a total LOS of 79 days for all incidents, followed by VIP with 38 days and IE with 30 (Appendix Table 5). The most frequent cause of all hospitalizations during OP therapy was neutropenic fever $(26.9 \%)$, followed by abdominal pain, cramps, or distension (11.5\%). The average IP stay for all 
Table 3 Incidents of grade 4 neutropenia reported by chemotherapy regimen and cycle

\begin{tabular}{|c|c|c|c|c|}
\hline \multirow[t]{2}{*}{ Patient Regimens } & \multicolumn{2}{|l|}{$\begin{array}{l}\text { Outpatient } \\
(n=22)\end{array}$} & \multicolumn{2}{|l|}{$\begin{array}{l}\text { Inpatient } \\
(n=16)\end{array}$} \\
\hline & $\begin{array}{l}\text { Incidence of grade } 4 \text { neutropenia } \\
\text { (number of patients) }\end{array}$ & $\begin{array}{l}\text { Cycle of therapy } \\
\text { ( } n=\text { incidents) }\end{array}$ & $\begin{array}{l}\text { Incidence of grade } 4 \text { neutropenia } \\
\text { (number of patients) }\end{array}$ & $\begin{array}{l}\text { Cycle of therapy } \\
\text { ( } n=\text { incidents) }\end{array}$ \\
\hline AIM & $14(9)$ & $\begin{array}{l}1(4) \\
2(6) \\
3(2) \\
4,6(1)\end{array}$ & $4(3)$ & $\begin{array}{l}1(3) \\
2(1)\end{array}$ \\
\hline TIP & $1(1)$ & $1(1)$ & $1(1)$ & $3(1)$ \\
\hline VIP & $4(2)$ & $\begin{array}{l}1(2) \\
2,3(1)\end{array}$ & $1(1)$ & $1(1)$ \\
\hline RICE & $1(1)$ & $2(1)$ & $8(5)$ & $\begin{array}{l}1(5) \\
2(2) \\
3(1)\end{array}$ \\
\hline IE & $10(8)$ & $\begin{array}{l}1(6) \\
2(2) \\
5,7(1)\end{array}$ & - & - \\
\hline AEWS1031 & $6(1)$ & $1-6(1)$ & - & - \\
\hline Carb/Doxo/Ifos & - & - & $5(1)$ & $1-5(1)$ \\
\hline ICE & - & - & $3(3)$ & $1(3)$ \\
\hline TI & - & - & $1(1)$ & $1(1)$ \\
\hline IVAC & - & - & $2(1)$ & $1,2(1)$ \\
\hline
\end{tabular}

*Regimens: AIM (doxorubicin, ifosfamide, mesna); TIP (paclitaxel, ifosfamide, cisplatin); VIP (etoposide, ifosfamide, cisplatin); R-ICE (rituximab, ifosfamide, carboplatin, etoposide); IE (ifosfamide, etoposide); AEWS 1031/1221 (vincristine, doxorubicin, cyclophosphamide, ifosfamide, etoposide); Carb/Doxo/Ifos (carboplatin, doxorubicin, ifosfamide); ICE (ifosfamide, carboplatin, etoposide); TI (paclitaxel, ifosfamide); IVAC (ifosfamide, etoposide, cytarabine)

OP hospitalizations due to an adverse reaction was 4.6 days (range 1-17); the longest duration was due to neutropenic fever, typhlitis, and E. coli bacteremia.

By transitioning to OP ifosfamide, the OP patient population avoided a total of 783 hospital bed days leading to a hospital cost savings of $\$ 2,103,921$. The IP therapies totaled 401 treatment days, which cost $\$ 1,077,487$ for IP hospital care in total. Additionally, the total number of OP versus IP labs was 634 and 804, respectively. There was an average of 3.5 labs ordered per OP treatment cycle and 10.9 labs per IP cycle. This led to an average cost of $\$ 336$ for labs per OP cycle and $\$ 1046$ per IP cycle with a cost savings of $\$ 710$ per cycle with the transition to OP therapy.

\section{Discussion}

Over the last decade, several centers have initiated Medicare and Medicaid Services (CMS) with alternative payment models in oncology addressing value-based care. One such program, the oncology care model (OCM), developed by the Center For Medicare and Medication Innovation, focuses on the larger spectrum of value-based metrics for oncology treatment $[18,19]$. These metrics have changed over the last couple of years; the goal is to reduce the cost of care while maintaining high-quality care in the oncology setting. This landmark pilot has brought about innovation, changes to access, enhanced affordability, and more OP care. Evaluating the use of IP chemotherapy for the OP setting was used to improve value-based care for OP treatment. The ability to reduce hospital bed utilization, minimize costs, and improve access for patient care has transformed our treatment strategy.

Ifosfamide utilization was addressed in 57 patients in the OP setting and 29 patients in the IP setting. Ifosfamide administration had more severe hematologic adverse drug effects (ADEs) occurring in the IP setting than the OP setting where treatment was found to be safe and welltolerated. OP ifosfamide therapy also provided a lower cost of care for patients and the institution. These findings were similar to previous studies from Coriat et al. (2009) and McBride et al. (2018). In these studies, OP ifosfamide therapy was shown to have a favorable toxicity profile, and the transition from the IP chemotherapy regimen to the OP setting resulted in decreased hospital bed utilization and increased cost savings [13, 20]. In the Corait et al. study, a single center gave 13 patients OP ifosfamide plus mesna therapy over 5 days every 21 weeks. They found evidence of clinical activity and a favorable toxicity profile with monotherapy ifosfamide. The total cost per cycle was $\$ 2133$ in an ambulatory setting versus $\$ 7642$ in the hospital [13], a reduction in cost by more than two-thirds when transitioning to the OP setting. The 
Fig. 2 a Toxicity incidence by OP chemotherapy cycle $(n=57)$. b Toxicity incidence by IP chemotherapy cycle $(n=29)$. *In $\mathbf{a}, \mathbf{b}$, other toxicities include edema, pain, headache, rash, mucositis, AKI, bleeding, tachycardia, hypotension, leukocytosis, and thrombocytosis. **Each incidence is per patient per cycle and not the accumulated records of ADEs each patient had overall within each cycle
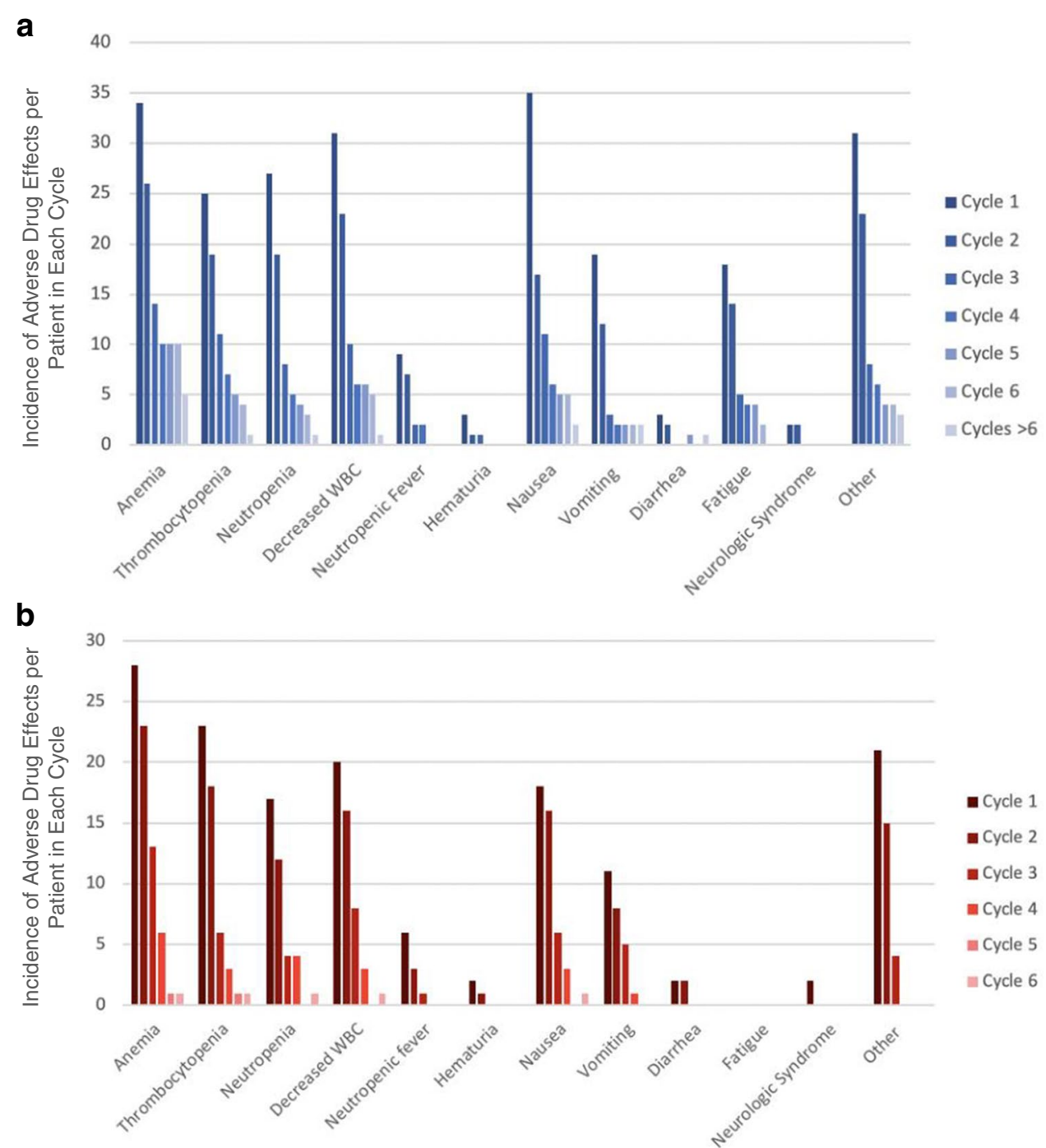

transition to OP chemotherapy led to favorable cost savings and improved models for ifosfamide treatment consistent with our findings.

The safety of this chemotherapy agent given in the OP was a concern for patients, caregivers, and nurses in evaluating this new model of care. A myriad of ifosfamide-containing treatment regimens was observed in our study. Many agents in these regimens were repeated both in the IP and OP aside from ifosfamide as seen in Table 1. In our OP chemotherapy regimens, the most commonly observed ADEs included anemia, leukopenia, and nausea, which were similar to other studies and regimens administered in the IP setting with ifosfamidecontaining regimens.

NF was seen in the IP setting more commonly than the OP (13.5\% vs. $10.9 \%$ of total cycles). In the OP setting, NF was seen in $11.5 \%$ of cycles with AIM and $9.8 \%$ with IE. The literature suggested a much higher rate with $31 \%$ and $53 \%$ with AIM and IE, respectively $[9,21]$. In our study, there were 23 patients (16 OP and 7 IP) with NF. Of the 20 total OP NF incidences, 3 were ungraded, 9 were grade 3, and 6 were grade 4 . Grade $4 \mathrm{NF}$ was found to have a higher incidence in the OP group (3.2\%) than the IP group (1.4\%). However, with the small numbers, it is not clear whether they are statistically different. Of the OP incidents, 19 lead to hospitalization while the remaining patient was hospitalized 3 days prior to NF secondary to other adverse effects. For many of the patients hospitalized, NF was the only reason for hospital admission. OP management of NF is an option for treatment as outlined in the American Society of Clinical Oncology (ASCO) and Infectious Diseases Society of America (IDSA) Practice Guidelines. The management of NF in the OP setting is based on clinical criteria and/or validated scoring tools (i.e., Multinational Association of Support Care in Cancer risk index and Clinical Index of Stable Febrile Neutropenia) [22].

Although $61 \%$ of OP patients were hospitalized at some point in their cancer treatment, a majority of the ADEs 
Table 4 Incidence of neutropenic fever

\begin{tabular}{|c|c|c|}
\hline \multirow[b]{2}{*}{ Chemotherapy type } & \multicolumn{2}{|c|}{ NF incidence } \\
\hline & $\begin{array}{l}\text { Outpatient } \\
(n=57)\end{array}$ & $\begin{array}{l}\text { Inpatient } \\
(n=29)\end{array}$ \\
\hline \multicolumn{3}{|c|}{ Type of ifosfamide regimen } \\
\hline AIM & 10 & 3 \\
\hline IE & 4 & - \\
\hline VIP & 3 & - \\
\hline TIP & 1 & - \\
\hline RICE & 1 & 3 \\
\hline AEWS1031 regimen A & 1 & - \\
\hline IVAC & - & 2 \\
\hline ICE & - & 1 \\
\hline Carb/Doxo/Ifos & - & 1 \\
\hline \multicolumn{3}{|l|}{ Incidence-based on cycle } \\
\hline Cycle 1 & 9 & 6 \\
\hline Cycle 2 & 7 & 3 \\
\hline Cycle 3 & 2 & 1 \\
\hline Cycle 4 & 2 & 0 \\
\hline Cycle 5 & 0 & 0 \\
\hline Cycle 6 & 0 & 0 \\
\hline Cycle $>6$ & 0 & 0 \\
\hline Bacterial Infection* & 6 & 4 \\
\hline \multicolumn{3}{|c|}{ Length of stay in hospital } \\
\hline $1-3$ days & 5 & 1 \\
\hline 4-6 days & 7 & 4 \\
\hline 7-9 days & 2 & 1 \\
\hline 10-12 days & 3 & 2 \\
\hline$>12$ days & 1 & 1 \\
\hline Unknown & 1 & $1 * *$ \\
\hline
\end{tabular}

*Bacterial infections for OP group included methicillin-resistant Staphylococcus epidermidis (MRSE) in urine, coccidioides mycosis, pan sensitive Escherichia coli, pan sensitive Klebsiella pneumoniae, pan sensitive Enterobacter cloacae and Clostridium difficile ( $C$ diff). Bacterial infections for IP group included: Klebsiella, Pseudomonas, Enterococcus faecium, and Escherichia coli

** Patient deceased shortly after NF status determined

${ }^{* * *}$ Regimens: Regimens: AIM (doxorubicin, ifosfamide, mesna); IE (ifosfamide, etoposide); VIP (etoposide, ifosfamide, cisplatin); TIP (paclitaxel, ifosfamide, cisplatin); R-ICE (rituximab, ifosfamide, carboplatin, etoposide); AEWS 1031 Regimen A (vincristine, doxorubicin, cyclophosphamide, ifosfamide, etoposide); IVAC (ifosfamide, etoposide, cytarabine); ICE (ifosfamide, carboplatin, etoposide); Carb/Doxo/Ifos (carboplatin, doxorubicin, ifosfamide)

documented in both the IP and OP settings were mild. Improving characterization of IP and OP ADE profiles allowed providers to better anticipate and improve the side effects management. For example, nausea has a high incidence in both IP and OP treatments, but it can typically be managed successfully with pharmacologic agents. Furthermore, ifosfamide has black box warnings for more uncommon but severe ADEs such as urotoxicity and neurotoxicity; in this study, the incidence by cycle of these ADEs was relatively low in both groups: hematuria (4.1\%, IP $n=3$ and $2.7 \%$, OP $n=5$ ) and neurologic syndrome requiring MB (2.7\%, IP $n=2$ and $2.2 \%$, OP $n=4)$. For those who experienced neurotoxicity, every patient recovered with MB treatment, suggesting this ADE could be handled on an OP basis. One patient was successfully rechallenged with ifosfamide therapy; the OP patient was initiated on AIM with ifosfamide dosing of $1500 \mathrm{mg} /$ $\mathrm{m}^{2}$ but suffered neurologic syndrome the second day of ifosfamide treatment. A month later, the patient was reinitiated with a shorter course of 3 treatment days and lower dose of ifosfamide at $1200 \mathrm{mg} / \mathrm{m}^{2}$. Dose reduction and shorter regimen in treatment days is a plausible solution for preventing future neurotoxicity. In Pelgrims et al.'s retrospective study, eight patients were treated with MB immediately upon clinical diagnosis. The recovery times for ifosfamide neurotoxicity were as follows: three patients within $12 \mathrm{~h}$, one within $24 \mathrm{~h}$, two after $48 \mathrm{~h}$, and two after $72 \mathrm{~h} \mathrm{[23].} \mathrm{Another} \mathrm{six} \mathrm{literature}$ reviews featuring MB treatment of ifosfamide neurotoxicity had 1 patient, each with varying ifosfamide regimens and MB dosing with varied recovery rates from 10 min to 8 days [24-29]. In addition to ifosfamide dose adjustment and/or shorter course of neurotoxicity prevention, it is also possible to pretreat with $\mathrm{MB}$ in patients with higher neurological risks such as chronic seizures (IP, $n=1$ ). Although this patient received treatment IP, pretreatment with $\mathrm{MB}$ still provides an option for prophylaxis in higher risk patients.

Using OP ifosfamide treatment substantially reduces the cost of administration. In the Corait et al. study, a patient's total cost per cycle for a $2-\mathrm{m}^{2}$ body surface area was $\$ 2133$ in an ambulatory setting versus $\$ 7642$ in the hospital; both include the costs of ifosfamide/mesna therapy and care in each setting for a 5-day infusion course [13]. In our OP setting, there was a reduction in the number of labs drawn and days spent in the hospital for daily monitoring. Minimization of expenses helps alleviate the financial burden for the healthcare system and insurance companies. This ambulatory infusion center saved $\$ 2,103,921$ over IP treatment. Additionally, the average cost of labs for frequent monitoring of IP treatment (\$1046) is triple the cost of OP treatment (\$336) per cycle. In retrospect, the frequency of labs will always have a higher cost in IP settings due to monitoring standards most hospitalized treatments require, which will skew ADRs and labs not observed but experienced in the outpatient setting. Assuming the cost of ifosfamide therapy and labs are equal in either setting, keeping patients out of the hospital has major cost savings and a clear role for ambulatory infusion centers to reduce healthcare system costs $[16,17]$. Although there are patients who require hospitalization for therapy, receiving OP cancer treatment has become more accessible. Patients may tolerate OP therapy as observed in this study; six IP patients had completed $4(n=4)$ to $5(n=2)$ cycles of OP 
therapy and had comparable ADE profiles. Transitioning those patients from IP AIM $(n=3)$ alone would have saved $\$ 290,196$. Ambulatory infusion centers have the capability to administer ifosfamide OP; they have the ability and supplies to correct moderate ADEs.

When reviewing colony-stimulating factors (CSF) in this study, the products primarily used were pegfilgrastim/pegfilgrastim-cbqv and filgrastim-sndz (31\% in IP; 7\% in OP). Over $90 \%$ of OP patients received pegfilgrastim products $20 \mathrm{~h}$ after chemotherapy since the majority of patients with mesna pumps were required to return to the clinic for the pump removal. In this study, IP patients received neulasta or neupogen in either IP/OP settings depending on their wellbeing and/or provider discretion; there were 7 patients who had stayed an extra day in the hospital to receive their CSF. Although pegfilgrastim products are not recommended for use between 14 days before or $24 \mathrm{~h}$ after chemotherapy administration, several studies, including our own, have shown no difference in outcomes [30-32]. Providing CSF within less than $24 \mathrm{~h}$ is possible, allowing an additional hospitalization day saved for both the institution and qualifying IP/OP patients.

Data supporting OP ifosfamide treatment as being efficacious, tolerable, and cost-effective could encourage insurance companies to consider OP therapy as a feasible and worthy option. If a patient's ifosfamide therapy meets the insurance treatment criteria such as able-bodied and a performance score (PS) of 0-1, OP treatment may be a viable alternative to IP. Should a patient warrant concerns of ifosfamide therapy, another option may be to administer therapy IP for the first cycle prior to committing to OP therapy which three IP patients in this study opted to do [11]. Transitioning ifosfamide to the OP setting provides a novel approach to treating patients while maximizing patient satisfaction and decreasing total patient care costs under an alternative payment model. With the COVID-19 pandemic overwhelming hospitals, the availability of outpatient therapies would free up inpatient beds for the crisis while safe-guarding patients with compromised immune systems undergoing active therapy. Additionally, the financial savings for an institution would help lower costs for the healthcare system and patients.

\section{Limitations}

There are many limitations in this study, which included the small sample size at a single institution and possible inaccuracy of cancer stage classification due to electronic medical record changes in 2017 or lack of documentation. Reported ADEs may not be solely associated with ifosfamide as patients were on combination therapies and may suffer from signs and/or symptoms due to progression of disease. Additionally, ADEs were not always described in detail in patients' electronic medical records, making the grading less standardizable. It is also reasonable IP patients who were hospitalized for therapy required close monitoring, which would increase ADEs reported for IP therapy. Consequently, the possibility of OP patients having the same number of ADEs/concerns as the IP counterpart is plausible since ADEs were recorded less frequently. When interpreting the data for ADEs, patients with more completed cycles or multiple ifosfamide therapies ( 1 in IP; 5 in OP) are overrepresented as these patients may have more recorded ADEs. Patient groups among ifosfamide regimens and of varying duration by treatment days were not differentiated either. Although not measured in this study, patients with better PS may be more likely treated in the OP setting since these patients would be able to tolerate chemotherapy outside of a hospital setting.

Another consideration to bear in mind is the comparison between IP and OP is not a 1:1 ratio. The disproportion of the therapies was often due to provider preference such as in the case of IP/OP lymphoma therapy or due to pharmacy transition of care in therapies for soft tissue sarcoma. Many providers at this institution opted for IP treatment over OP treatment since there were standards already in place for IP chemotherapies; only in the last several years, some providers and pharmacists were able to advocate for certain chemotherapies to be moved to OP such as riuximab [20]. In our study, not all therapies are generalizable to all patients receiving ifosfamide treatment. OP patients were also an average of 10 years older than the IP patients which may skew certain outcomes as well and the differences in malignancies are not duplicated in either arm such as OP treatment in patients with lung cancers or soft tissue sarcomas whereas patients with lymphoma were treated IP. Furthermore, the population of IP was small and several did not complete their therapy, which lowers the ability to accurately analyze tolerance for IP ifosfamide therapy. High-risk individuals with increased baseline risk and differing health care needs between the groups were not placed in a separate comparison group in this study. Lastly, it is very important to note the costs garnered in this study are not accurate to the institution but rather likely costs found from a state adjusted hospital indicator so the savings are estimates.

\section{Conclusions}

Transitioning to OP ifosfamide therapy is feasible for academic and community infusion centers with the administration of ifosfamide OP being safe, well-tolerated, and associated with decreased total cost of care. While the frequency of ADEs is quite diverse in IP and OP settings, outpatient ifosfamide administration lowers costs for patients and the institution, by and large in the reduction of both hospital stay days and aggressive lab tests. Ifosfamide OP therapy has not only practical application in monetary saving but also 
in sparing hospital rooms for patients who do require any kind of IP treatments such as the current situation with the COVid-19 pandemic. Although ifosfamide OP regimens are relatively recent, there are protocols available to an oncology team on how to administer ifosfamide in an ambulatory setting properly and defining procedures in which anticipated ADEs would be handled appropriately. Providers and pharmacists can transition certain patients' ifosfamide therapy to OP setting and monitor for side effects while lowering healthcare costs for the patient and institution.

\section{Appendix 1}

Table 5 Incidence of hospital visits by cause and the length of stay based on OP chemotherapy

\begin{tabular}{ll}
\hline Cause of hospitalization & Incidence (\% cycles per regimen) \\
\hline Neutropenic fever & 19 \\
Abdominal pain/cramps/distention & 6 \\
Neutropenia & 3 \\
E. coli bacteremia & 2 \\
Pseudomonas bacteremia & 1 \\
Salmonella bacteremia & 1 \\
C. difficile & 1 \\
Altered mental status & 3 \\
Chest pain & 3 \\
Tachycardia & 1 \\
Dehydration & 1 \\
Pain & 2 \\
Afib & 2 \\
Intractable nausea/vomiting & 3 \\
Rectal bleeding & 1 \\
Neutropenic typhlitis & 1 \\
Bilateral pulmonary embolism & 1 \\
Neurotoxicity & 1 \\
Hemorrhagic cystitis & 1 \\
Hematuria & 1 \\
Pneumonia & 1 \\
Leg pain/swelling & 1 \\
Inadequate oral intake/nutrition & 1 \\
Chemotherapy type & Total LOS (days) \\
AIM & 79 \\
IE & 30 \\
VIP & 38 \\
TIP & 16 \\
RICE & 16 \\
IGEV & 0 \\
Afosfamide alone & \\
AEWS 1221 Regimen A & 1031 Regimen A \\
\hline
\end{tabular}

\section{Appendix 2}

*Other adverse effects include: hematuria, edema, pain, headache, rash, mucositis, AKI, bleeding, tachycardia, hypotension, leukocytosis, and thrombocytosis.

\section{Appendix 3. Overall toxicity - incidences for OP therapy (total $=582$ )}

Table 6 Overall toxicity-incidences for OP therapy (total $=582$ )

\begin{tabular}{llllll}
\hline Adverse effect & Grade 1 & Grade 2 & Grade 3 & Grade 4 & Ungraded \\
\hline Neutropenia & 7 & 8 & 15 & 36 & \\
$\begin{array}{l}\text { Febrile neutro- } \\
\text { penia }\end{array}$ & - & - & 11 & 6 & 3 \\
$\quad$ Thrombocytopenia & 27 & 18 & 10 & 17 & \\
Anemia & 48 & 34 & 21 & 8 & \\
Neurotoxicity & - & - & - & - & 4 \\
Hematuria & & & & & 5 \\
Nausea & 66 & 12 & 2 & 0 & \\
Vomiting & 31 & 11 & 1 & 0 & \\
Diarrhea & 2 & 5 & 0 & 0 & \\
Fatigue & 30 & 12 & 3 & 0 & \\
Others* & - & - & - & - & 134 \\
\hline
\end{tabular}

Author contribution Study design: AM, CB, AA, AO and KN; data analysis: $\mathrm{EE}, \mathrm{HB}, \mathrm{AK}, \mathrm{ARB}, \mathrm{DP}$ and $\mathrm{AO}$; interpretation of data and critical revision of the manuscript for important intellectual content: all authors.

Availability of data and material N/A.

Code availability N/A.

\section{Declarations}

Ethics approval. N/A

Consent to participate. N/A

Consent for publication. All the authors consent to publication.

Competing interests The authors declare no competing interests.

\section{References}

1. Dollinger M (1996) Guidelines for hospitalization for chemotherapy. Oncologist 1:107-111

2. Foster AE, Reeves DJ (2017) Inpatient antineoplastic medication administration and associated drug costs: institution 
of a hospital policy limiting inpatient administration. PT 42:388-393

3. Boyle DA. The Rising Cost of Cancer Drugs Takes a Toll. Oncology Nursing News. https://www.oncnursingnews.com/web-exclu sives/the-rising-cost-of-cancer-drugs-takes-a-toll. Published January 4, 2019. Accessed April 13, 2020.

4. Hong SJ, Li EC, Matusiak LM, Schumock GT (2018) Spending on antineoplastic agents in the United States, 2011 to 2016. J Oncol Practice 14(11):e683-e691

5. Tran G, Zafar SY (2018) Financial toxicity and implications for cancer care in the era of molecular and immune therapies. Ann Trans Med. 6:166. https://doi.org/10.21037/atm.2018.03.28

6. Knight TG, Deal AM, Dusetzina SB et al (2018) Financial toxicity in adults with cancer: adverse outcomes and non-compliance. $\mathrm{J}$ Oncol Practice. https://doi.org/10.1200/JOP.18.00120

7. Peters E, Schulz LM, Reuss-Borst M (2016) Quality of life after cancer- how the extent of impairment is influenced by patient characteristics. BMC Cancer 16:787

8. Oosterom AT, Mouridsen HT et al (2002) Results of randomised studies of the EORTC Soft Tissue and Bone Sarcoma Group (STBSG) with two different ifosfamide regimens in first- and second-line chemotherapy in advanced soft tissue sarcoma patients. Eur J Cancer 38:2397-2406

9. Patel SR, Vadhan-Raj S, Burgess MA et al (1998) Results of two consecutive trials of dose-intensive chemotherapy with doxorubicin and ifosfamide in patients with sarcomas. Am J Clin Oncol 21:317-321

10 Pico JL, Rosti G, Kramar A et al (2005) Genito-Urinary Group of the French Federation of Cancer Centers; EBMT. A randomised trial of high-dose chemotherapy in the salvage treatment of patients failing first-line platinum chemotherapy for advanced germ cell tumours. Ann Oncol 16:1152-9

11. Ifex. Prescribing information. Baxter International Inc. March 2012.

12. Mesnex. Prescribing information. Baxter International Inc. March 2014.

13. Coriat R, Mir O, Camps S et al (2009) Ambulatory administration of 5-day infusion ifosfamide mesna: a pilot study in sarcoma patients. Cancer Chemother Pharmacol 65(3):491-495. https:// doi.org/10.1007/s00280-009-1054-1

14. COVID-19 Patient Care Information. ASCO. https://www.asco. org/asco-coronavirus-information/care-individuals-cancer-duringcovid-19. Published April 10, 2020. Accessed April 13, 2020.

15. Common Terminology Criteria for Adverse Events (CTCAE) Version 5. National Institutes of Health. https://ctep.cancer.gov/proto coldevelopment/electronic_applications/docs/CTCAE_v5_Quick_ Reference_5x7.pdf. Published November 27, 2017. Accessed April 27, 2020.

16. Hospital Adjusted Expenses per Inpatient Day. The Henry J. Kaiser Family Foundation. https://www.kff.org/health-costs/stateindicator/expenses-per-inpatient-day. Published February 21, 2020. Accessed April 2, 2020.

17. Laboratory Pricing. Sparrow Health System. 2020.

18. American Society of Clinical Oncology (2016) The State of Cancer Care in America, 2016: a report by the American Society of Clinical Oncology. Journal of Oncology Practice 12(4):339-383

19. Clough JD, Kamal AH (2015) Oncology care model: short- and long-term considerations in the context of broader payment reform. J Oncol Pract 11:319-332

20. Mcbride A, Campen CJ, Camamo J et al (2018) Implementation of a pharmacy-managed program for the transition of chemotherapy to the outpatient setting. Am J Health Syst Pharm 75(9):e246e258. https://doi.org/10.2146/ajhp170138

21. Sasada S, Kodaira M, Shimoi T et al (2016) Ifosfamide and etoposide chemotherapy in the treatment of recurrent/refractory rhabdomyosarcoma in adults. Anticancer Res 36(5):2429-2432
22. Taplitz RA, Kennedy EB, Bow EJ et al (2018) Outpatient management of fever and neutropenia in adults treated for malignancy: American Society of Clinical Oncology and Infectious Diseases Society of America Clinical Practice Guideline Update. J Clin Oncol 36:1443-1453. https://doi.org/10.1200/JCO.2017.77.6211

23. Pelgrims J, Vos FD, Brande JVD, Schrijvers D, Prové A, Vermorken JB (2000) Methylene blue in the treatment and prevention of ifosfamide-induced encephalopathy: report of 12 cases and a review of the literature. Br J Cancer 82(2):291-294. https://doi. org/10.1054/bjoc.1999.0917

24. Küpfer A, Aeschlimann C, Wermuth B, Cerny T (1994) Prophylaxis and reversal of ifosfamide encephalopathy with methyleneblue. The Lancet 343(8900):763-764. https://doi.org/10.1016/ s0140-6736(94)91839-2

25. Zulian GB, Tullen E, Maton B (1995) Methylene blue for ifosfamide-associated encephalopathy. N Engl J Med 332(18):12391240. https://doi.org/10.1056/nejm199505043321817

26. Ferrero JM, Eftekari P, Largillier R, Dreyfus G, Namer M (1995) Traitement d'une encéphalopathie à l'ifosfamide par le bleu de méthylène. Bull Cancer 82:598-599

27. Demandt M, Wandt H (1996) Erfolgreiche Behandlung von Ifosfamid-bedingten zentralnervösen Nebenwirkungen mit Methylenblau. Dtsch Med Wochenschr 121:575

28. Alonso J, Nieto Y, López J, Martín M, Díaz-Rubio E (1996) Ifosfamide encephalopathy and methylene-blue: a case report. Ann Oncol 7(6):643-644. https://doi.org/10.1093/oxfordjournals. annonc.a010686

29. Koschuth A, Späth-Schwalbe E, Possinger K (1996) Methylenblau bei Ifosfamid-induzierter Enzephalopathie. Dtsch Med Wochenschr 121:1210

30. Burris HA, Belani CP, Kaufman PA, et al. Pegfilgrastim on the same day versus next day of chemotherapy in patients with breast cancer, non-small-cell lung cancer, ovarian cancer, and nonhodgkin's lymphoma: results of four multicenter, double-blind, randomized phase II studies. Journal of oncology practice. https:// www.ncbi.nlm.nih.gov/pmc/articles/PMC2868638/. Published May 2010. Accessed April 3, 2020.

31. Neulasta. Prescribing Information. Amgen Inc. 2002 to 2015.

32. Eckstrom J, Bartels T, Abraham I et al (2019) A single-arm, retrospective analysis of the incidence of febrile neutropenia using same-day versus next-day pegfilgrastim in patients with gastrointestinal cancers treated with FOLFOX or FOLFIRI. Support Care Cancer 27:873-878

33. Le Cesne A, Judson I, Crowther D, Rodenhuis S, Keizer HJ, Van Hoesel Q, Blay JY, Frisch J, Van Glabbeke M, Hermans C, Van Oosterom A, Tursz T, Verweij J (2000) Randomized phase III study comparing conventional-dose doxorubicin plus ifosfamide versus high-dose doxorubicin plus ifosfamide plus recombinant human granulocyte-macrophage colony-stimulating factor in advanced soft tissue sarcomas: A trial of the European Organisation for Research and Treatment of Cancer/Soft Tissue and Bone Sarcoma Group. J Clin Oncol 18(14):2676-2684

34. Miser JS, Kinsella TJ, Triche TJ et al (1987) Ifosfamide with mesna uroprotection and etoposide: an effective regimen in the treatment of recurrent sarcomas and other tumors of children and young adults. J Clin Oncol 5(8):1191-1198. https://doi.org/10. 1200/jco.1987.5.8.1191

35. Van Winkle P, Angiolillo A, Krailo M, Cheung YK, Anderson B, Davenport V, Reaman G, Cairo MS, Children's Cancer Group (2005) Ifosfamide, carboplatin, and etoposide (ICE) reinduction chemotherapy in a large cohort of children and adolescents with recurrent/refractory sarcoma: the Children's Cancer Group (CCG) experience. Pediatr Blood Cancer 44(4):338-47

36. Nichols CR, Catalano PJ, Crawford ED, Vogelzang NJ, Einhorn LH, Loehrer PJ (1998) Randomized comparison of cisplatin and etoposide and either bleomycin or ifosfamide in treatment of 
advanced disseminated germ cell tumors: an Eastern Cooperative Oncology Group, Southwest Oncology Group, and Cancer and Leukemia Group B Study. J Clin Oncol 16(4):1287-1293

37. Hinton S, Catalano PJ, Einhorn LH, Nichols CR, David Crawford E, Vogelzang N, Trump D, Loehrer PJ Sr (2003) Cisplatin, etoposide and either bleomycin or ifosfamide in the treatment of disseminated germ cell tumors: final analysis of an intergroup trial. Cancer 97(8):1869-1875

38. Kondagunta GV, Bacik J, Donadio A, Bajorin D, Marion S, Sheinfeld J, Bosl GJ, Motzer RJ (2005) Combination of paclitaxel, ifosfamide, and cisplatin is an effective second-line therapy for patients with relapsed testicular germ cell tumors. J Clin Oncol 23(27):6549-6555

39. Kurobe M, Kawai K, Oikawa T, Ichioka D, Kandori S, Takaoka E, Kojima T, Joraku A, Suetomi T, Miyazaki J, Nishiyama H (2015) Paclitaxel, ifosfamide, and cisplatin (TIP) as salvage and consolidation chemotherapy for advanced germ cell tumor. J Cancer Res Clin Oncol 141(1):127-133 (Epub 2014 Jul 26)

40. Santoro A, Magagnoli M, Spina M, Pinotti G, Siracusano L, Michieli M, Nozza A, Sarina B, Morenghi E, Castagna L, Tirelli
U, Balzarotti M (2007) Ifosfamide, gemcitabine, and vinorelbine: a new induction regimen for refractory and relapsed Hodgkin's lymphoma. Haematologica 92(1):35-41

41. Magagnoli M, Spina M, Balzarotti M, Timofeeva I, Isa L, Michieli M, Capizzuto R, Morenghi E, Castagna L, Tirelli U, Santoro A (2007) IGEV regimen and a fixed dose of lenograstim: an effective mobilization regimen in pretreated Hodgkin's lymphoma patients. Bone Marrow Transplant 40(11):1019-1025 (Epub 2007 Oct 1)

42. Zinzani PL, Broccoli A, Gioia DM, Castagnoli A, Ciccone G, Evangelista A, Santoro A, Ricardi U, Bonfichi M, Brusamolino E, Rossi G, Anastasia A, Zaja F, Vitolo U, Pavone V, Pulsoni A, Rigacci L, Gaidano G, Stelitano C, Salvi F, Rusconi C, Tani M, Freilone R, Pregno P, Borsatti E, Sacchetti GM, Argnani L, Levis A (2016) Interim positron emission tomography response-adapted therapy in advanced-stage Hodgkin lymphoma: final results of the phase II part of the HD0801 study. J Clin Oncol 34(12):13761385 (Epub 2016 Feb 16)

Publisher's note Springer Nature remains neutral with regard to jurisdictional claims in published maps and institutional affiliations. 